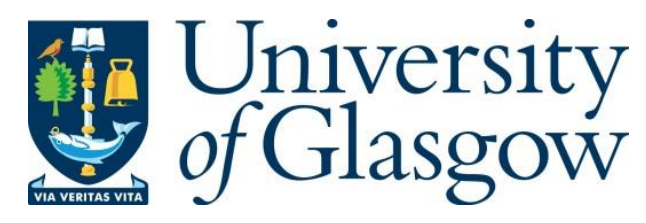

da Silva, F. E., Iaremczuk, A. L. V., Souza, R. D., Brante, G., Moritz, G. L. and Hussain, S. (2020) Hybrid ARQ in wireless packetized predictive control. IEEE Sensors Letters, (doi: 10.1109/LSENS.2020.2986835)

There may be differences between this version and the published version. You are advised to consult the publisher's version if you wish to cite from it.

http://eprints.gla.ac.uk/214617/

Deposited on: 23 April 2020

Enlighten - Research publications by members of the University of Glasgow http://eprints.gla.ac.uk 


\title{
Hybrid ARQ in Wireless Packetized Predictive Control
}

\author{
Fernando Effting da Silva ${ }^{1}$, Ana Lúcia Vieira laremczuk², Richard Demo Souza ${ }^{1}$, Glauber Brante ${ }^{2}$, \\ Guilherme Luiz Moritz², Sajjad Hussain ${ }^{3}$ \\ ${ }^{1}$ Federal University of Santa Catarina, Brazil, fernando.engen@gmail.com, richard.demo@ufsc.br \\ ${ }^{2}$ Federal University of Technology - Paraná,Brazil, ana.iaremczuk@gmail.com,gbrante@utfpr.edu.br, moritz@utfpr.edu.br \\ 3 University of Glasgow, United Kindom, sajjad.hussain@glasgow.ac.uk
}

\begin{abstract}
Motivated by new wireless applications that rely on ultra-reliable low latency communications, while supporting the transmission of short packets, we introduce a method that reduces the wireless resources consumption for real-time control of physical systems. Leveraging the tight interaction between control and communication systems, we make use of packetized predictive control along with incremental redundancy hybrid automatic repeat request, aiming at minimizing the energy consumption of a packet by optimizing the transmit power and prediction length of the controller. Our results show that the proposed strategy can save up to $45 \%$ of wireless resources when compared to a state-of-the-art method.
\end{abstract}

Index Terms-Sensor system networks, sensor buses and communications, networkable sensors-actuators

\section{INTRODUCTION}

The number of sensors and devices connected to wireless networks is expected to keep growing in the following years, enabling several new applications [1]. A key related emerging technology is known as Wireless Networked Control Systems (WNCS) [2], which plays a major role in the Industry 4.0 paradigm [3]. Transmission failures or deadline misses may affect the WNCS performance. Achieving ultrareliable low latency communications (URLLC) [4] is considerably challenging in wireless sensor networks, and even more complicated in the case of short transmission packets [5], which is the typical traffic generated by sensors and actuators within WNCS. Moreover, the usual separate communication and control design is suboptimal, leading to the demand for communication and control co-design [6].

In [2] the authors present an exhaustive review on WNCS and codesign interactions between communication and control for optimal performance. They discuss the impact of several variables, like sampling period, packet delay, dropout, communications protocol, and energy consumption. Moreover, the authors in [7] minimize the energy consumption per bit, for a given WCNS. After determining the maximal allowed outage probability, which is a function of the control system stability, they adapt the bit rate and the transmit power. Their results show that the energy consumption depends on the controller as well as on the communication system. The authors in [8] propose an optimal resource allocation scheme in search of maximizing the spectral efficiency in URLLC for real-time control systems. The end result is a system with optimal resource allocation while still providing the necessary control performance.

In terms of reducing the wireless resources needed to meet the reliability requirement in a WNCS, the authors in [6] made a significant progress. They consider the use of Packetized Predictive Control (PPC) [9], in which a controller forecasts future commands that are sent to an actuator, so that commands sent over the wireless link contain not only those for the current time slots, but also for future time slots. This provides the system with the ability to withstand a given packet loss without becoming unstable. By working out the power and time-frequency resource allocation, as previously detailed by the same authors in [10], they find the optimal prediction length of the controller that leads to the lowest resources consumption. Surprisingly, higher wireless traffic, a consequence of transmitting predicted control commands, may lead to savings in wireless resources.

Another technique that provides an efficient use of wireless resources is Hybrid Automatic Repeat reQuest (HARQ) [11]. In this case a feedback channel is used to inform the transmitter about the packet decoding status at the receiver. When transmission failures occur, additional packets with replicas or increased redundancy are transmitted to improve the decoding probability. Since most of the time retransmissions are not needed, only the necessary resources are used to attain the required reliability. However, HARQ must be carefully designed when latency constraints must be met. In this regard, the work in [12] evaluates the performance of HARQ under latency constraints, where the retransmissions are replicas of the original transmissions and are combined at the receiver, in a scenario representative of smart grid teleprotection applications. Results show significant energy savings, however, the focus of [12] is not the case of WNCS, and therefore there is no communication-control co-design.

In this paper, we aim at minimizing the wireless resources consumption of a WNCS making use of PPC, as in [6], but considering the application of incremental redundancy HARQ. Therefore, instead of using all the available channel uses for transmitting a packet with the control commands, we consider the transmission of an initial shorter packet conveying the control commands, therefore with increased error probability, but that saves a considerable amount of wireless resources. In case of failure, additional redundancy is sent in a second transmission, as to guarantee the required reliability within the latency budget. Thus, this paper addresses the matter of obtaining the optimal prediction length of the controller, utilizing short blocklength packets along with HARQ, as to achieve even lower wireless resources consumption than in [6], [10]. Numerical results show that we can save as much as $45 \%$ of the resources in some scenarios when compared to [6], [10].

The rest of this paper is as follows. In Section II we present the system model and the problem formulation. In Section III we discuss the proposed strategy and its optimization. In Section IV we present numerical results, while Section V concludes the paper.

\section{SYSTEM MODEL}

We assume the control system in Fig. 1, containing: $i$.) a wired link between Sensor and Controller, which is assumed perfectly reliable; and ii.) a wireless link between Controller and Actuator, which is prone to errors. Then, in the sequel we define the PPC model, the finite block-length framework and the problem formulation. 


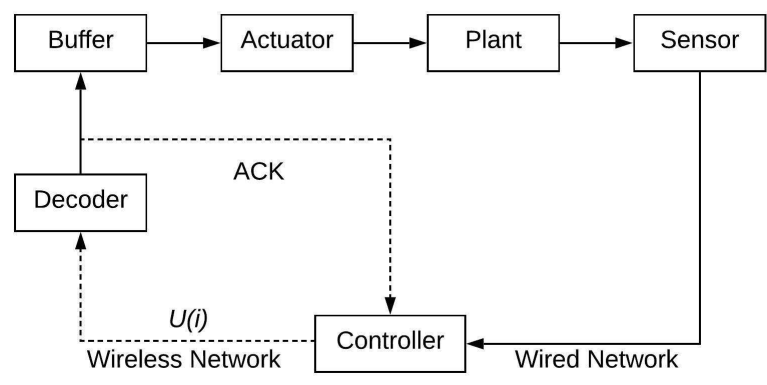

Figure 1. Sensor and Controller are connected by a wired link, while the Controller and the Actuator communicate through a wireless link.

\begin{tabular}{|c|c|c|c|}
\hline Head ( $H$ bits) & \multicolumn{2}{|c|}{ Payload (K.L bits) } & \\
\hline & $u_{1}(i)(L$ bits $)$ & $u_{2}(i)(L$ bits $)$ & $u_{\kappa}(i)(L$ bits $)$ \\
\hline
\end{tabular}

Figure 2. Packet structure in the case of finite block-length.

\section{A. PPC Model}

In PPC, the sensor observes the current plant output and then transmits it to the controller, which in turn predicts the plant state and calculates a control sequence

$$
\mathbf{U}(i)=\left[u_{1}(i), u_{2}(i), \cdots, u_{K}(i)\right],
$$

where $i$ is the index of the time slot and $K$ is the prediction length (in control commands). Hence, $u_{1}(i)$ is the control command for the current $i$-th time slot, and $\left[u_{2}(i), \cdots, u_{K}(i)\right]$ are the predicted control commands for the $K-1$ future time slots. It is the Decoder/Buffer function to feed the Actuator with the most suitable control command $u_{1}(i)$ at the start of each time slot. In the case of an outage event, the Decoder may ask for a retransmission, if there is enough time for processing the request, otherwise, the previously cached commands may be used until the wireless communication is reestablished.

Therefore, the Buffer acts as a safety measure against packet outages, so that the system may tolerate up to $K-1$ sequential packet losses before running out of control commands to execute in the next time slot. Such event is termed control outage in this paper, whose probability $p_{c}$ depends on the the packet error probability $p_{e}$ (which in turn depends on the wireless link). Then, to attain a certain control outage probability we must guarantee that [10]:

$$
p_{e} \leq p_{c}^{\frac{1}{K}} .
$$

\section{B. Finite Blocklength Analysis}

From asymptotic information theory, we are able to transmit a given information block with arbitrarily low error probability if the number of channel uses $n$ can be made sufficiently large [11]. However, such assumption of sufficiently large blocklength is not necessarily practical in the case of delay sensitive applications like WNCS. Considering $B$ the system bandwidth and $T$ the time slot duration (which also determines the control period), the transmitter can only send $n=B T$ symbols while meeting the latency constraint.
When the system parameters impose small values of $n$ for meeting the latency requirement, classical theory can not be applied and the non-asymptotic finite block-length analysis [5] should be be used.

Moreover, in the case of short packets, the size of the metadata becomes comparable to the size of the payload, and therefore must be taken into account. For instance, Fig. 2 illustrates the case of the control system under study, where the payload of each packet is composed by $H$ bits of header and $K$ control commands, each with $L$ bits. Therefore, the total number of bits in a single packet is [10]:

$$
N=H+K L .
$$

Then, the maximum achievable rate $R\left(n, p_{e}\right)$ in bits per symbols (or per channel use) of the communication system is a function of the blocklength $n$ and the packet error probability $p_{e}$, so that [5]

$$
R\left(n, p_{e}\right) \approx C-\sqrt{\frac{V}{n}} Q^{-1}\left(p_{e}\right)+\frac{\log _{2} n}{2 n},
$$

where $Q^{-1}$ is the inverse $Q$-function, the channel capacity is

$$
C=\log _{2}(1+\gamma),
$$

$\gamma$ is the signal-to-noise ratio (SNR), and the channel dispersion is

$$
V=\gamma \frac{2+\gamma}{(1+\gamma)^{2}}\left(\log _{2} e\right)^{2} \approx\left(\log _{2} e\right)^{2},
$$

where the approximation is valid for sufficiently high SNR.

Moreover, the SNR at the Decoder is

$$
\gamma=\frac{P G}{N_{0} B},
$$

where $P$ is the transmission power, $N_{0}$ is the noise power spectral density of the additive white Gaussian noise (AWGN), and $G$ represents the channel gain (i.e. the path loss).

We can also represent the time-frequency resources used by each packet, in symbols or channel uses, as

$$
n=\frac{N}{R\left(n, p_{e}\right)},
$$

where $1 \leq n \leq n_{\max }$ to meet the control deadline $(T)$.

Combining (4) and (8), the packet error probability in the case of finite blocklength can be written as

$$
p_{e} \approx Q\left(\frac{n C-N+\frac{\log _{2} n}{2}}{\sqrt{n V}}\right) .
$$

\section{Problem Formulation}

It is the goal of this paper to minimize the transmission energy consumption of a packet, which can be written as

$$
E=P T=\frac{P}{B} n=\frac{P}{B} \cdot \frac{H+K L}{R\left(n, p_{e}\right)} .
$$

Combining (2), (5), (9) and (10) our optimization problem becomes

$$
\begin{array}{rl}
\min _{K, P, n} & E=\frac{P}{B} \cdot \frac{H+K L}{R\left(n, p_{e}\right)}, \\
\text { s.t. } & p_{e}=Q\left(\frac{n C-N+\frac{\log _{2} n}{2}}{\sqrt{n V}}\right) \leq p_{c}^{\frac{1}{K}}, \\
& 1 \leq n \leq n_{\max } .
\end{array}
$$

This problem was discussed in [10] for the case without HARQ, in which the optimal power $P^{\star}$ and blocklength $n^{\star}$ are

$$
P^{\star}=\left(2 \frac{1}{2 n^{\star}} \sqrt{n^{\star}} Q^{-1}\left(p_{c}^{\frac{1}{K}}\right) \log _{2}(e)+N-\frac{1}{2} \log _{2}\left(n^{\star}\right)-1\right) \frac{N_{0} B}{G},
$$



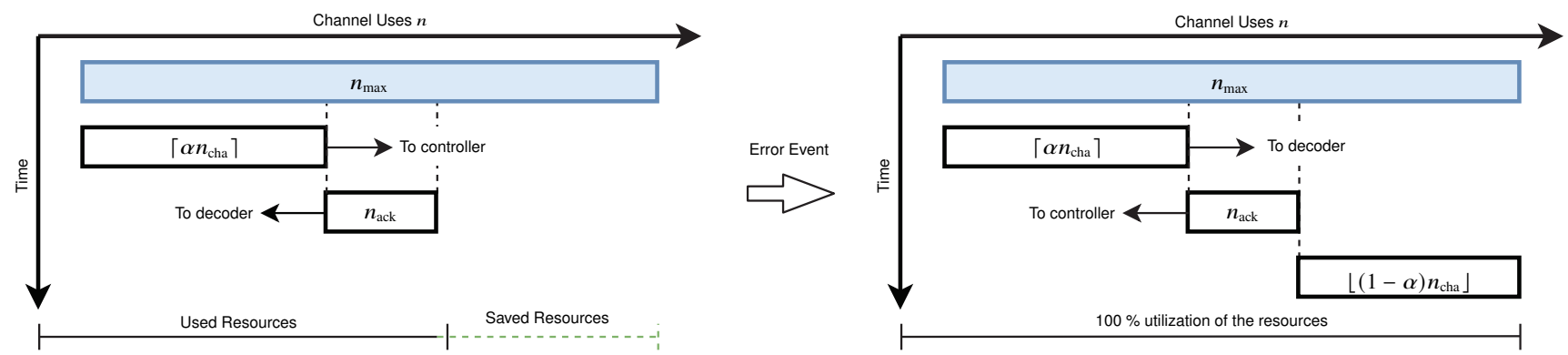

Figure 3. The proposed method consists in transmitting a frame using $\left\lceil\alpha n_{\text {cha }}\right\rceil$ channel uses for the first transmission attempt, with $0<\alpha \leq 1$. In the event of a decoding error, incremental redundancy is transmitted making use of the rest of the available $\left\lfloor(1-\alpha) n_{\text {cha }}\right\rfloor$ channel uses.

$$
n^{\star}=n_{\max } .
$$

If there is a feedback channel between Decoder and Controller, then HARQ can be applied, which in turn can further lower the overall energy consumption, as we describe in the sequel.

\section{PROPOSED METHOD}

In [6], [10] all the available $n_{\max }$ channel uses within the latency constraint are spent in a single transmission. Instead, in this work we propose the use of HARQ with incremental redundancy. At each time slot the Controller attempts a first transmission using just a fraction of the available channel uses. Therefore, we use an increased rate in this first transmission attempt when compared to [6], [10], since we send the same information but in less channel uses. We assume that the Decoder always sends a feedback message which indicates whether the first transmission could be correctly decoded or not. At this point, if the Controller can correctly decode the feedback message as an ACK, it can save energy by switching off its transmitter during the rest of the time slot.

However, in case the Decoder is unable to successfully receive the initial attempt, or if the ACK message is not correctly decoded by the Controller, a complementing packet with incremental redundancy is sent using the remaining channel uses within the latency deadline.

In the proposed method $n_{\text {max }}=n_{\text {cha }}+n_{\text {ack }}$, where $n_{\text {cha }}$ is the total number of channel uses to be employed in the Controller to Decoder link and $n_{\text {ack }}$ is the number of symbols in the ACK message. Next, we assume that the available channel uses are divided between two transmissions whose size are determined by an arbitrary constant $\alpha$, where $0<\alpha \leq 1$. In the first transmission, $\left\lceil\alpha n_{\text {cha }}\right\rceil$ symbols are sent, while the remaining $\left\lfloor(1-\alpha) n_{\text {cha }}\right\rfloor$ symbols may be used in a potential retransmission ${ }^{1}$, as illustrated in Fig. 3.

\section{A. Energy Savings using $H A R Q$}

The first step to evaluate the energy savings of the proposed method is using (12) to calculate the required power $P_{\text {ir }}$ to meet the control outage probability $p_{c}$ after $n_{\text {cha }}$ channel uses. We can observe that $P_{\text {ir }}>P^{\star}$ in (12) since $n_{\text {cha }}<n_{\max }$. Next, when using $P_{\text {ir }}$ in the first transmission attempt, but with $\left\lceil\alpha n_{\text {cha }}\right\rceil$ channel uses, the error probability is $p_{e, 1}>p_{e}$, which can be calculated from (9) with

${ }^{1}$ Throughout this paper we have considered the case of a single retransmission due to the scenario being latency constrained, and therefore multiple retransmissions would required excessive data rates per transmission attempt. $n=\left\lceil\alpha n_{\text {cha }}\right\rceil$. A retransmission is requested by the Decoder with probability $p_{e, 1}$. Thus, the Controller has a $\left(1-p_{e, 1}\right)$ probability of saving energy by not transmitting during the $\left\lfloor(1-\alpha) n_{\text {cha }}\right\rfloor$ remaining channel uses if the first transmission attempt already succeeded.

Moreover, we should not neglect that the feedback channel is also prone to errors, and therefore there is a probability $p_{e, \text { ack }}$ that the ACK message is not received by the Controller. In this case, a retransmission is performed even when not requested. With this information, we can write the total energy consumption of the proposed scheme as

$$
\begin{aligned}
E_{\mathrm{ir}}= & {\left[\left(E_{1}+E_{\mathrm{ack}}\right)\left(1-p_{e, 1}\right)+\left(E_{1}+E_{2}+E_{\mathrm{ack}}\right) p_{e, 1}\right]\left(1-p_{e, \text { ack }}\right) } \\
& +\left(E_{1}+E_{2}+E_{\mathrm{ack}}\right) p_{e, \mathrm{ack}},
\end{aligned}
$$

where $E_{1}=\frac{P_{\text {ir }}\left[\alpha n_{\text {cha }}\right]}{B}$ is the energy consumption of the first transmission attempt, $E_{2}=\frac{P_{\text {ir }}\left\lfloor(1-\alpha) n_{\text {cha }}\right\rfloor}{B}$ is the energy consumption of the retransmission, while $E_{\text {ack }}=\frac{P_{\text {ack }} n_{\text {ack }}}{B}$ is the energy consumption of the ACK message.

\section{NUMERICAL RESULTS}

In this section we compare the proposed scheme with the method in [6], [10]. We assume $N_{0}=-204 \mathrm{dBW} / \mathrm{Hz}$, channel gain $G=$ $-80 \mathrm{~dB}$, a header of $H=32$ bits, control command length of $L=8$ bits, $n_{\text {ack }}=10$ symbols, $P_{\text {ack }}$ is equal to the value of $P_{\text {ir }}$ when $K=1$, and a conservative ${ }^{2} p_{e, \text { ack }}=10^{-2}$. In addition, following [10], [14], the target control outage probability is set to $p_{c}=10^{-9}$.

Fig. 4 shows the energy consumption $(E)$ versus the prediction length of the controller $(K)$. We compare the proposed method with [6], [10], for $n_{\max }=250$. The energy consumption of the proposed scheme is lower for every considered value of $K$. Moreover, the optimal $K$ is smaller for the proposed method, decreasing from $K=5$ to $K=2$, with the energy consumption being around $34.5 \%$ less than that of [6], [10] for their optimal $K$. Such analysis is complemented by Fig. 5, when $n_{\max }=500$, in which the proposed scheme is $45 \%$ more efficient than the method in [6], [10]. In addition, the optimal prediction length is $K=1$ for this case, which indicates that HARQ alone is a better strategy than predictive control when the latency constraints are relaxed.

Furthermore, the optimal $\alpha$ as a function of $K$ is shown in Fig. 6 for $n_{\max }=\{250,500\}$, where we observe that when the prediction length

${ }^{2}$ The finite blocklength formulation in (9) is not accurate for very small information blocklengths [13], and therefore, instead of using (9) with $k=1$ and $n_{\text {ack }}=10$, we set a very conservative value of $p_{e, \text { ack }}=10^{-2}$. In the ACK message there is only one bit of information, and therefore the data rate is very low, what should make the link naturally very robust. 


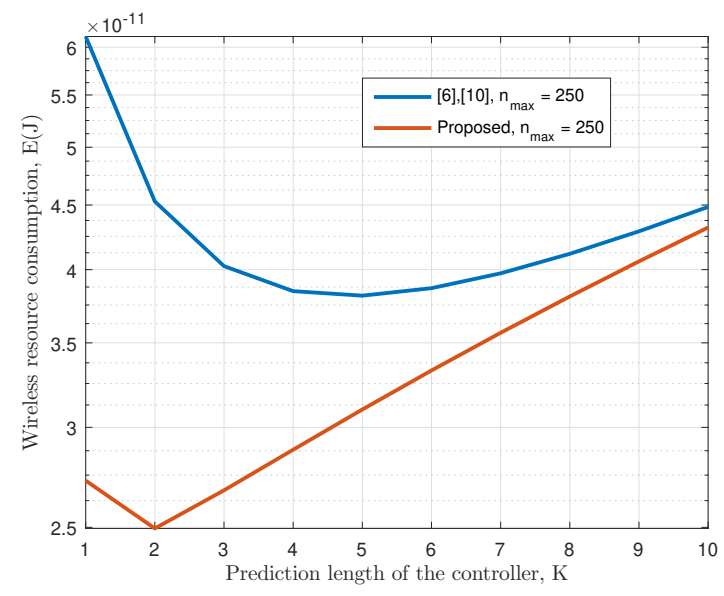

Figure 4. Energy consumption versus $K$ for the proposed method and that of [6], [10], with $n_{\max }=250$.

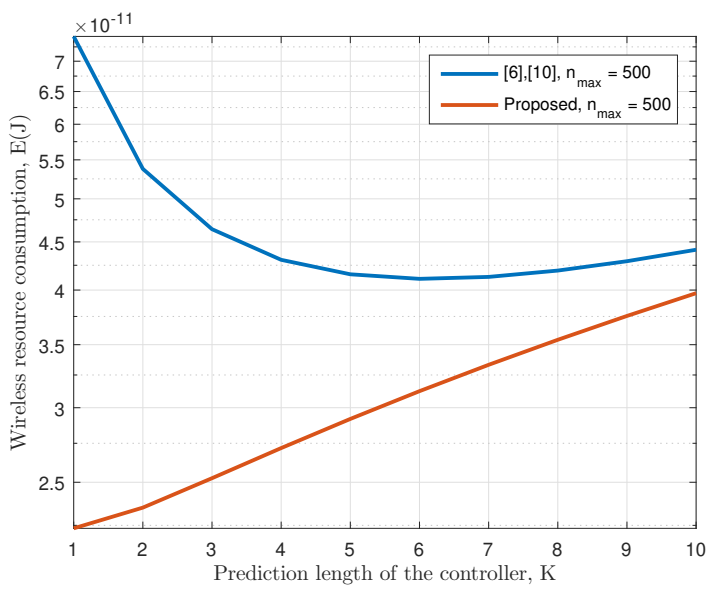

Figure 5. Energy consumption versus $K$ for the proposed method and that of [6], [10], with $n_{\max }=500$.

increases, more channel uses are required in the first transmission to keep the outage probability low enough so that it does not compromise the energy efficiency. For small values of $K$, the optimal strategy becomes to transmit with a small fraction of the available channel uses within the latency constraint. Under these circumstances, the first transmission already yields in a reasonable low outage probability. For instance, $p_{e}=8.7 \%$ when $n_{\max }=250$, while $p_{e}=3.4 \%$ when $n_{\max }=500$. Therefore in more than $90 \%$ of the time a retransmission is not needed, and more than half of the time-frequency resources can be saved, yielding the energy savings of the proposed scheme.

\section{CONCLUSION}

We proposed a resource allocation method to minimize the energy consumption in WNCS based on PPC and HARQ with incremental redundancy. By the optimization of transmit power and prediction length of the controller, we were able to reduce the energy consumption with respect to the baseline scheme by as much as $45 \%$.

\section{ACKNOWLEDGMENT}

This work was partially supported in Brazil by FAPESC (201813170), CNPq, CAPES Finance Code 001, and CAPES PrInt Program \#698503P.

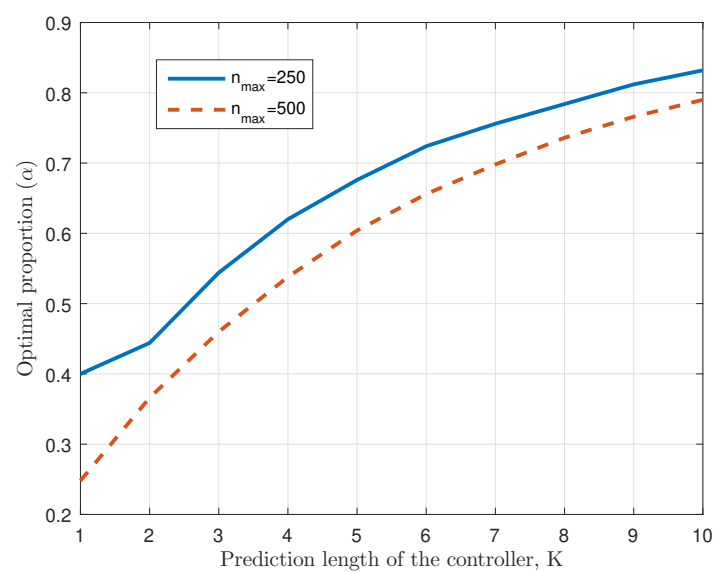

Figure 6. Optimal $\alpha$ as a function of $K$ for $n_{\max }=\{250,500\}$.

\section{REFERENCES}

[1] V. Cisco, "Cisco visual networking index: Forecast and trends, 2017-2022," White Paper, vol. 1, 2018.

[2] P. Park, S. C. Ergen, C. Fischione, C. Lu, and K. H. Johansson, "Wireless network design for control systems: A survey," IEEE Communications Surveys \& Tutorials, vol. 20, no. 2, pp. 978-1013, 2017.

[3] B. Chen, J. Wan, L. Shu, P. Li, M. Mukherjee, and B. Yin, "Smart factory of industry 4.0: Key technologies, application case, and challenges," IEEE Access, vol. 6, pp. 6505-6519, 2017.

[4] P. Popovski, J. J. Nielsen, C. Stefanovic, E. De Carvalho, E. Strom, K. F. Trillingsgaard, A.-S. Bana, D. M. Kim, R. Kotaba, J. Park et al., "Wireless access for ultra-reliable low-latency communication: Principles and building blocks," IEEE Network, vol. 32, no. 2, pp. 16-23, 2018.

[5] G. Durisi, T. Koch, and P. Popovski, "Toward massive, ultrareliable, and low-latency wireless communication with short packets," Proceedings of the IEEE, vol. 104, no. 9, pp. 1711-1726, 2016.

[6] G. Zhao, M. A. Imran, Z. Pang, Z. Chen, and L. Li, "Toward real-time control in future wireless networks: communication-control co-design," IEEE Communications Magazine, vol. 57, no. 2, pp. 138-144, 2018

[7] R. Scaciota, G. L. Moritz, G. Brante, and R. D. Souza, "Minimization of energy consumption per bit using an average dwell-time approach for wireless networked control systems," IEEE Access, vol. 7, pp. 81 839-81 848, 2019.

[8] B. Chang, L. Zhang, L. Li, G. Zhao, and Z. Chen, "Optimizing resource allocation in URLLC for real-time wireless control systems," IEEE Transactions on Vehicular Technology, vol. 68, no. 9, pp. 8916-8927, 2019.

[9] Q. Wang, Y. Zou, and Y. Niu, "Event-triggered model predictive control for wireless networked control systems with packet losses," in IEEE International Conference on Cyber Technology in Automation, Control, and Intelligent Systems, 2015, pp. $1281-1286$

[10] X. Tong, G. Zhao, M. A. Imran, Z. Pang, and Z. Chen, "Minimizing wireless resource consumption for packetized predictive control in real-time cyber physical systems," in IEEE International Conference on Communications Workshops, 2018, pp. 1-6.

[11] A. Goldsmith, Wireless Commun. USA: Cambridge Univ. Press, 2005.

[12] J. P. Battistella Nadas, O. Onireti, R. D. Souza, H. Alves, G. Brante, and M. A. Imran, "Performance analysis of hybrid ARQ for ultra-reliable low latency communications," IEEE Sensors Journal, vol. 19, no. 9, pp. 3521-3531, May 2019.

[13] Y. Polyanskiy, H. V. Poor, and S. Verdú, "Channel coding rate in the finite blocklength regime," IEEE Transactions on Information Theory, vol. 56, no. 5, p. 2307, 2010.

[14] M. Luvisotto, Z. Pang, and D. Dzung, "Ultra high performance wireless control for critical applications: Challenges and directions," IEEE Transactions on Industrial Informatics, vol. 13, no. 3, pp. 1448-1459, 2017. 